\title{
Vectorial scanning force microscopy using a nanowire sensor
}

\author{
Nicola Rossi1, Floris R. Braakman1, Davide Cadeddu1, Denis Vasyukov1, Gözde Tütüncüoglu², \\ Anna Fontcuberta i Morral² and Martino Poggio ${ }^{1 \star}$
}

\begin{abstract}
Self-assembled nanowire (NW) crystals can be grown into nearly defect-free nanomechanical resonators with exceptional properties, including small motional mass, high resonant frequency and low dissipation. Furthermore, by virtue of slight asymmetries in geometry, a NW's flexural modes are split into doublets oscillating along orthogonal axes. These characteristics make bottom-up grown NWs extremely sensitive vectorial force sensors. Here, taking advantage of its adaptability as a scanning probe, we use a single NW to image a sample surface. By monitoring the frequency shift and direction of oscillation of both modes as we scan above the surface, we construct a map of all spatial tip-sample force derivatives in the plane. Finally, we use the NW to image electric force fields distinguishing between forces arising from the NW charge and polarizability. This universally applicable technique enables a form of atomic force microscopy particularly suited to mapping the size and direction of weak tip-sample forces.
\end{abstract}

A tomic force microscopy (AFM) $)^{1,2}$ exists in several forms and is now routinely used to image a wide variety of surfaces, in some cases with atomic ${ }^{3}$ or sub-atomic resolution ${ }^{4}$. Owing to its versatility, this technique has found application in fields such as solid-state physics, materials science, biology and medicine. Variations on the basic technique, including contact and noncontact modes, allow it to be applied under diverse conditions and with enhanced contrast for specific target signals. The measurement of multiple mechanical harmonics yields information on the nonlinearity of the tip-sample interaction, while monitoring higher mechanical modes provides further types of imaging contrast. Today, these various types of AFM are most often carried out using cantilevers that are processed by top-down methods from crystalline Si and are hundreds of micrometres long, tens of micrometres wide and of the order of $1 \mu \mathrm{m}$ thick.

In recent years, researchers have developed new types of mechanical transducers that are fabricated by bottom-up processes ${ }^{5}$. These resonators are built molecule-by-molecule in processes that are typically driven by self-assembly or directed self-assembly. Prominent examples include doubly clamped carbon nanotubes $(\mathrm{CNTs})^{6}$, suspended graphene sheets ${ }^{7}$ and NW cantilevers ${ }^{8-13}$. Assembly from the bottom up allows for structures with extremely small masses and low defect densities. A small motional mass both enables the detection of atomic-scale adsorbates and results in high mechanical resonance frequencies, decoupling the resonators from common sources of noise. Near structural perfection results in low mechanical dissipation and therefore high thermally limited force sensitivity. These factors result in extremely sensitive mechanical sensors: for example, CNTs have demonstrated yoctogram mass resolution ${ }^{14}$ and a force sensitivity down to a few $\mathrm{zN} \mathrm{Hz}^{-1 / 2}$ at cryogenic temperatures ${ }^{15,16}$.

Nevertheless, given their extreme aspect ratios and their ultrasoft spring constants, both CNTs and graphene resonators are extremely difficult to apply in scanning probe applications. NWs on the other hand, when arranged in the pendulum geometry, that is, with their long axes perpendicular to the sample surface, are well-suited as scanning probes as their orientation prevents the tip from snapping into contact ${ }^{17}$. When brought close to a surface, NWs experience extremely low non-contact friction ${ }^{18}$ making near-surface $(<100 \mathrm{~nm})$ force sensitivities around $1 \mathrm{aN} \mathrm{Hz}^{-1 / 2}$. As a result, NWs have been used as force transducers in nuclear magnetic resonance force microscopy ${ }^{19}$ and may be amenable to other ultrasensitive microscopies such as Kelvin probe force microscopy ${ }^{20}$ or for the spectroscopy of small friction forces ${ }^{21}$. Furthermore, their highly symmetric cross-section results in orthogonal flexural mode doublets that are nearly degenerate $\mathrm{e}^{10,13}$. In the pendulum geometry, these modes can be used for the simultaneous detection of in-plane forces and spatial force derivatives along two orthogonal directions ${ }^{22}$. Although one-dimensional (1D) dynamic lateral force microscopy can be realized using the torsional mode of conventional AFM cantilevers ${ }^{23-27}$, the ability to simultaneously image all vectorial components of nanoscale force fields is of great interest. Not only would it provide more information on tipsample interactions, but it would also enable the investigation of inherently $2 \mathrm{D}$ effects, such as the anisotropy or non-conservative character of specific interaction forces.

Here, we use individual as-grown NWs to realize the vectorial scanning force microscopy of a patterned surface. By monitoring the first-order flexural mode doublet of the NWs we fully determine the magnitude and direction of the static tip-sample force derivatives in the $2 \mathrm{D}$ scanning plane. As a proof-of-principle, we also map the force field generated by voltages applied to a sample with multi-edged gate electrodes and identify the contributions of the NW charge and polarizability to tip-sample interactions.

\section{NW force sensors}

The GaAs/AlGaAs NWs studied here are grown by molecular beam epitaxy, as described in the Methods. They have a predominantly zinc-blende crystalline structure and display a regular hexagonal cross-section. The NWs are grown perpendicular to the Si growth substrate and remain attached to it during the measurements to maintain good mechanical clamping and to avoid the introduction of defects through processing. Both of these factors help to minimize mechanical dissipation. Measurements are performed with the NWs enclosed in 

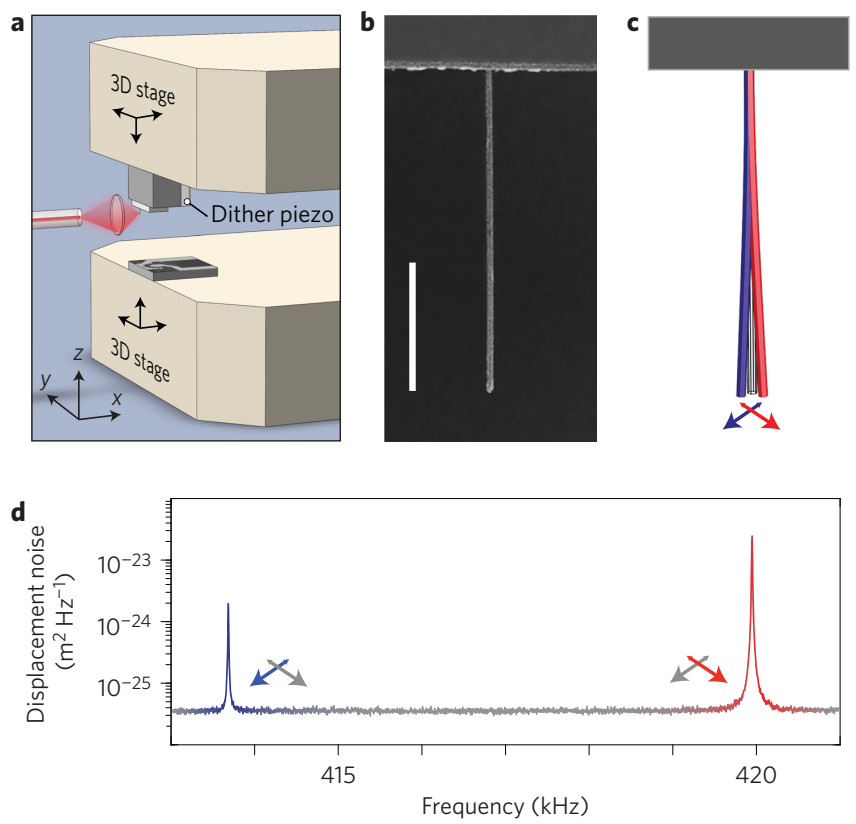

Figure 1 | Experimental set-up. a, Schematic drawing of the measurement set-up. A fibre-optic interferometer is aligned with a single NW using a piezoelectric positioning stage (top). A second stage (bottom) is used to position and scan the sample surface under the NW. $\mathbf{b}$, A SEM image of NW1, a typical GaAs/AIGaAs NW. Scale bar, $10 \mu \mathrm{m}$. c, A schematic diagram showing the two orthogonal fundamental flexural modes of the NW.

d, The displacement spectral noise density of the fundamental mode doublet measured by fibre-optic interferometry for NW1. We extract $\theta_{0}=15^{\circ}$.

an ultra-high vacuum chamber at the bottom of a liquid ${ }^{4} \mathrm{He}$ bath cryostat with a base temperature of $4.2 \mathrm{~K}$ and pressure of $10^{-7} \mathrm{mbar}$. The experimental set-up allows us to interferometrically monitor the displacement of single NWs as we scan them above a sample surface, as shown in Fig. 1a and described in the Methods. In the presented measurements, we use two individual NWs, one of which is shown in the scanning electron micrograph (SEM) in Fig. 1b, however, similar results were obtained from several other NWs. When required, the mechanical motion of the NW can be driven using a piezoelectric transducer fixed to the back of the NW chip holder.

The NWs can be characterized by their displacement noise spectral density. Here, the measured mechanical response is driven by the Langevin force resulting from the coupling between the NW and the thermal bath. As shown in Fig. 1d, NW1 shows two distinct resonance peaks at $f_{1}=414 \mathrm{kHz}$ and $f_{2}=420 \mathrm{kHz}$, corresponding to the two fundamental flexural eigenmodes polarized along two orthogonal directions (see schematic in Fig. 1c). The modes are split by $\delta=6.26 \mathrm{kHz}$ and have nearly identical quality factors of $Q_{i}=5 \times 10^{4}$, as determined by both ring-down measurements and by fitting the thermal noise spectral density with that of a damped harmonic oscillator. The splitting between the modes is many times their linewidths, a property observed in several measured NWs. Finite element modelling has shown that even small crosssectional asymmetries $(<1 \%)$ or clamping asymmetries can lead to mode splittings similar to those observed ${ }^{28}$.

We resolve the two first-order flexural modes with different signal-to-noise ratios, given that the principal axes $\hat{\boldsymbol{r}}_{1}$ and $\hat{\boldsymbol{r}}_{2}$ of the modes are rotated by some angle $\theta_{0}$ with respect to the optical detection axis $\hat{\boldsymbol{x}}^{10}$. Although we only measure NW displacement projected along $\hat{x}$, displacement along other directions in the $x-y$ plane are also accessible by aligning the interferometer beam slightly off-centre with respect to the NW's central axis ${ }^{22,29}$. Experimentally we find that when the optical detection axis is aligned with the central axis of the NWs, thermal motion in a direction
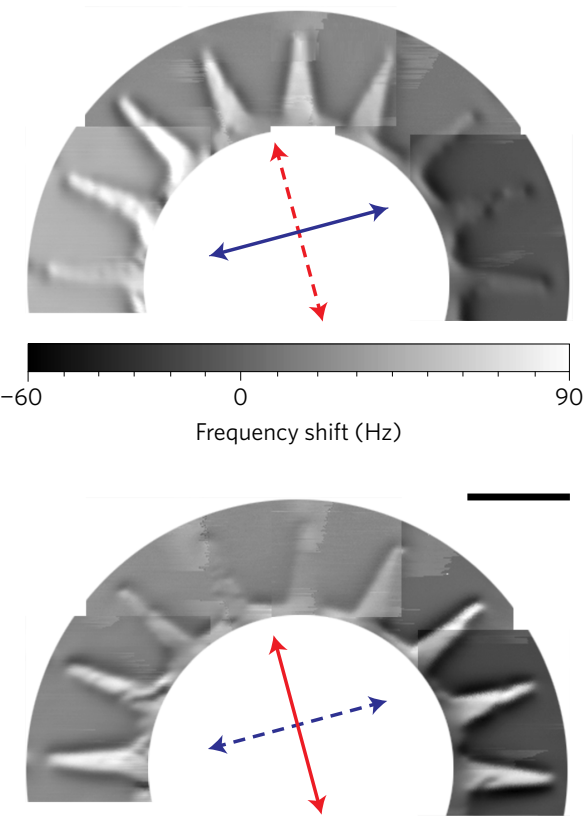

Figure $\mathbf{2}$ | Mode 1 and mode $\mathbf{2}$ frequency shift images. The top (bottom) panel shows $\Delta f_{1}(x, y)\left(\Delta f_{2}(x, y)\right)$ of $N W 1$, which has $\theta_{0}=15^{\circ}$, at a distance of $70 \mathrm{~nm}$ from a pattern of Au finger gates on a Si substrate. The greyscale is proportional to the respective frequency shifts and the blue (red) arrows show the directions $\hat{\boldsymbol{r}}_{1}\left(\hat{\boldsymbol{r}}_{2}\right)$ of mode 1 (2) as determined by thermal noise measurements far from the surface. Scale bar, $5 \mu \mathrm{m}$.

perpendicular to the detection axis falls below our detection sensitivity. Hence, the total measured displacement is $x(t)=r_{1}(t) \sin \theta_{0}+r_{2}(t) \cos \theta_{0}$, where $r_{1}$ and $r_{2}$ represent the displacement of each flexural mode. The mean square displacement generated by uncorrelated thermal noise is then $\left\langle x^{2}\right\rangle=P_{1}+P_{2}$, where $P_{1}=\left\langle r_{1}^{2}\right\rangle \sin ^{2} \theta_{0}$ and $P_{2}=\left\langle r_{2}^{2}\right\rangle \cos ^{2} \theta_{0}$ represent the integrated powers of each measured resonance in the spectral density. Given that the motional mass $m$ of the two orthogonal flexural modes is the same, using the equipartition theorem we find the ratio of their mean-square thermal displacements $\left\langle r_{1}^{2}\right\rangle /\left\langle r_{2}^{2}\right\rangle=f_{2}^{2} / f_{1}^{2}$. Therefore, from the measured thermal peaks in the spectral density, we calculate the angle $\theta_{0}=\arctan \left(\left(f_{1} / f_{2}\right) \sqrt{P_{1} / P_{2}}\right)$ between $\hat{\boldsymbol{r}}_{1}\left(\hat{\boldsymbol{r}}_{2}\right)$ and $\hat{\boldsymbol{x}}(\hat{\boldsymbol{y}})$. Furthermore, since the spring constants of each flexural mode are given by $k_{i}=k_{\mathrm{B}} T /\left\langle r_{i}^{2}\right\rangle$, where $i=1,2$ and $k_{\mathrm{B}}$ is the Boltzmann constant, we obtain values that are typically on the order of $10 \mathrm{mN} \mathrm{m}^{-1}$. These parameters yield mechanical dissipations $\Gamma_{i}=k_{i} /\left(2 \pi f_{i} Q_{i}\right)$ and thermally limited force sensitivities $S_{F_{i}}^{1 / 2}=\sqrt{4 k_{\mathrm{B}} T \Gamma_{i}}$ around $100 \mathrm{pg} \mathrm{s}^{-1}$ and $5 \mathrm{aN} \mathrm{Hz}^{-1 / 2}$, respectively.

\section{Two-mode scanning probe microscopy}

To use the NW as a scanning probe, we approach the sample and scan it in a plane below the NW tip. By monitoring the mechanical properties of the NW-that is, the frequency, dissipation and orientation of its doublet modes-we image the sample topography via the tip-sample interaction. Such microscopy can be accomplished by measuring the NW thermal displacement spectral density as the sample surface is scanned below it. Although such a measurement provides a full mechanical characterization of the modes, it is time-consuming due to the small displacement. A technique that is more amenable to fast spatial scans uses the resonant excitation of the doublet modes through two independent phase-locked loops to track both frequencies simultaneously (see Methods).

Figure 2 shows the frequency shifts $\Delta f_{1}(x, y)$ and $\Delta f_{2}(x, y)$ of the doublet modes as a sample is scanned below the tip of NW1. The sample consists of nine 5 - $\mu \mathrm{m}$-long and 200 -nm-thick finger gates of $\mathrm{Au}$ on a $\mathrm{Si}$ substrate that are radially disposed and equally 


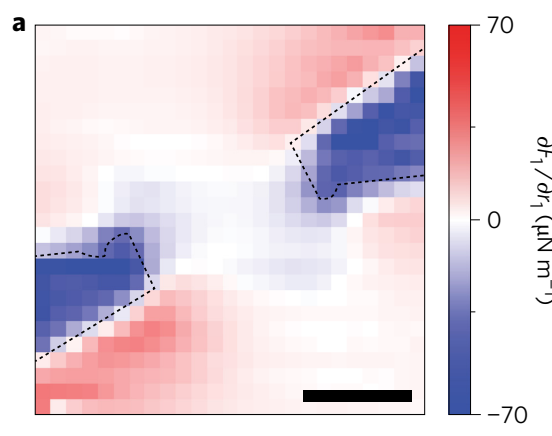

d
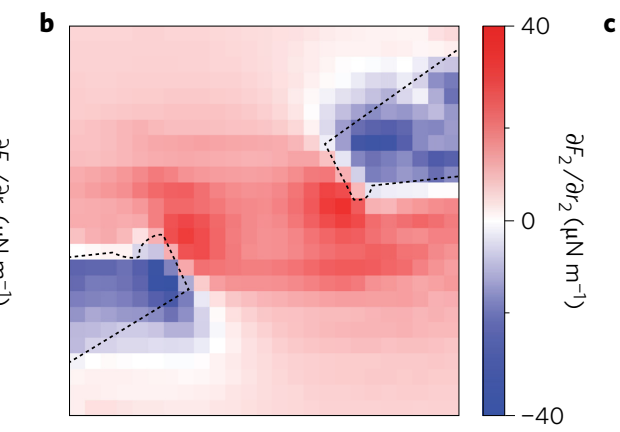

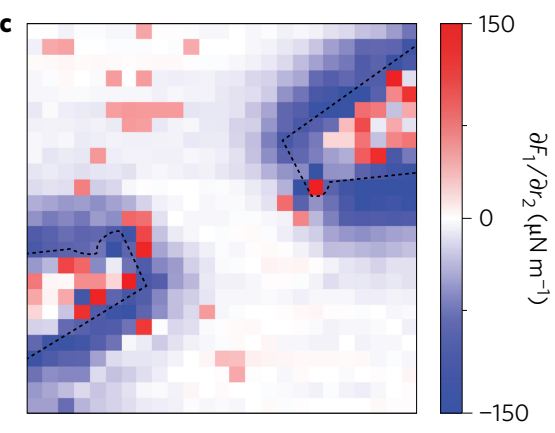

e

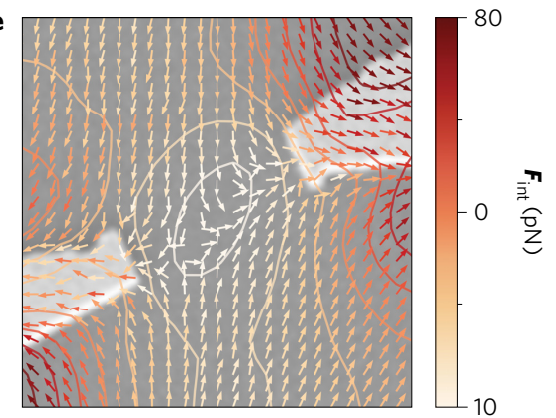

ș

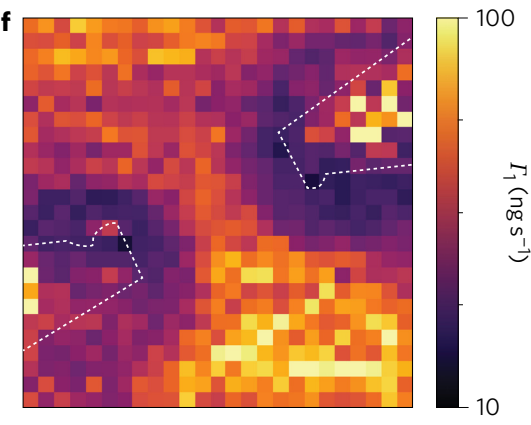

g

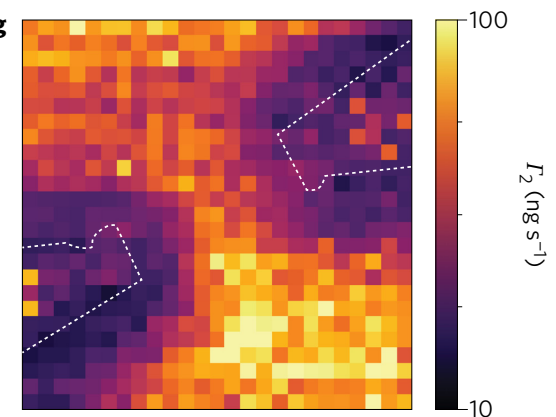

Figure 3 | 2D tip-sample force derivative, force and dissipation images. a-c, Force derivatives $F_{11}(\mathbf{a}), F_{22}(\mathbf{b})$ and $F_{12}=F_{21}(\mathbf{c})$, respectively, extracted from thermal noise data as a function of the $x-y$ position of NW2 $70 \mathrm{~nm}$ over the gated sample. The dashed lines indicate the edges of the finger gates, as obtained by SEM. Scale bar, $1 \mu \mathrm{m}$. d , Schematic picture of the direction of the flexural eigenmodes at two tip-sample distances $z$. The grey axes indicate the directions of the unperturbed axes with $\theta_{0}=46^{\circ}$. The eigenmodes rotate through an angle $\phi$ when brought closer to the sample above a given point $(x, y)$. e, In-plane tip-sample force field $\boldsymbol{F}_{\text {int }}$. The map is numerically integrated from the force derivatives up to a spatially constant force. The solid lines show the contours of constant force magnitude. $\mathbf{f}, \mathbf{g}$, Dissipation $\Gamma_{i}$ of each mode as a function of the $x-y$ position.

spaced along a semicircle (see Supplementary Information for an SEM of the sample). The finger gates and their tapered shape are intended to provide edges at a variety of different angles, highlighting the directional sensitivity of the orthogonal modes. The measurement in Fig. 2 is performed using the phase-locked loops with an oscillation amplitude of $6 \mathrm{~nm}$. The spatial scan is performed at a distance of $70 \mathrm{~nm}$ from the Au surfaces without feedback to maintain a constant tip-sample separation, that is, in 'open-loop'. The Au gates are grounded during the measurement.

The frequency shift images clearly delineate the topography of the patterned sample, with each mode showing stronger contrast for features aligned along orthogonal directions. These two directions (identified by noting the direction of the fingers with maximum contrast) agree with the angle $\theta_{0}=15^{\circ}$ measured for NW1 via the thermal noise shown in Fig. 1d. Edges (that is, large topographical gradients) that point perpendicular (parallel) to the mode oscillation direction appear to produce the strongest (weakest) contrast. Tip-sample interactions producing the frequency shifts in non-contact AFM can include electrostatic, van der Waals or chemical bonding forces depending on the distance. In our case, because of the large spacing, they are dominated by electrostatic forces.
To understand the measurements, we describe the motion of the NW tip in each of the two fundamental flexural modes as a driven damped harmonic oscillator:

$$
m \ddot{r}_{i}+\Gamma_{i} \dot{r}_{i}+k_{i} r_{i}=F_{\mathrm{th}}+F_{i}
$$

where $m$ is the effective mass of the fundamental flexural modes, $F_{\text {th }}$ is the Langevin force, $F_{i}$ is the component of the tip-sample force along $\hat{\boldsymbol{r}}_{i}$, and $i=1,2$. Following the treatment of Gloppe et al. ${ }^{22}$ and expanding $F_{i}$ for small oscillations around the equilibrium $r_{i}=0$, we have $F_{i} \approx F_{i}(0)+r_{j} \partial F_{i} /\left.\partial r_{j}\right|_{0}$. By replacing this expansion in equation (1), we find that this tip-sample interaction produces new doublet eigenmodes with modified spring constants $k_{i}^{\prime}$ (see Supplementary Information):

$$
\begin{aligned}
k_{1,2}^{\prime}= & \frac{1}{2}\left[k_{1}+k_{2}-F_{11}-F_{22}\right. \\
& \left. \pm \sqrt{\left(k_{1}-k_{2}-F_{11}+F_{22}\right)^{2}+4 F_{12} F_{21}}\right]
\end{aligned}
$$

where we use a shorthand notation for the force derivatives $F_{i j} \equiv \partial F_{i} /\left.\partial r_{j}\right|_{0}$. In addition to inducing frequency shifts, tip-sample force derivatives with non-zero shear components $\left(F_{i j} \neq 0\right.$ for $\left.i \neq j\right)$ 
a
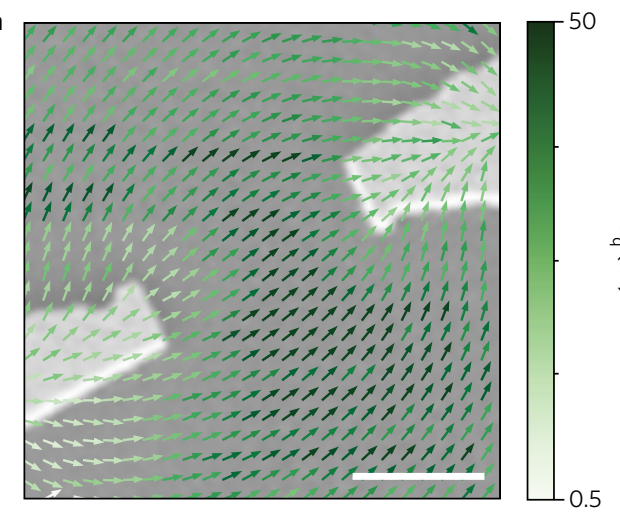

C

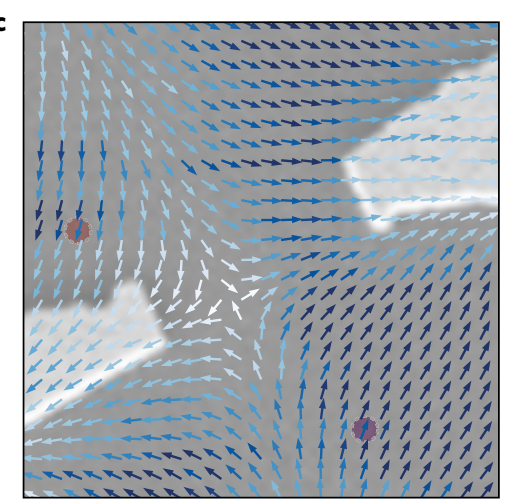

b

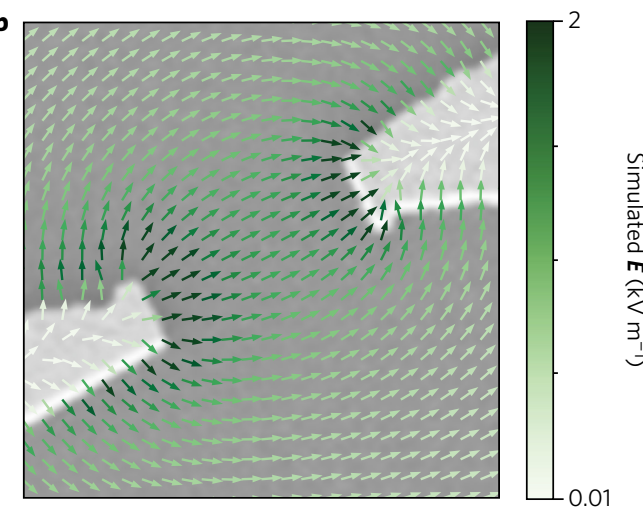

d

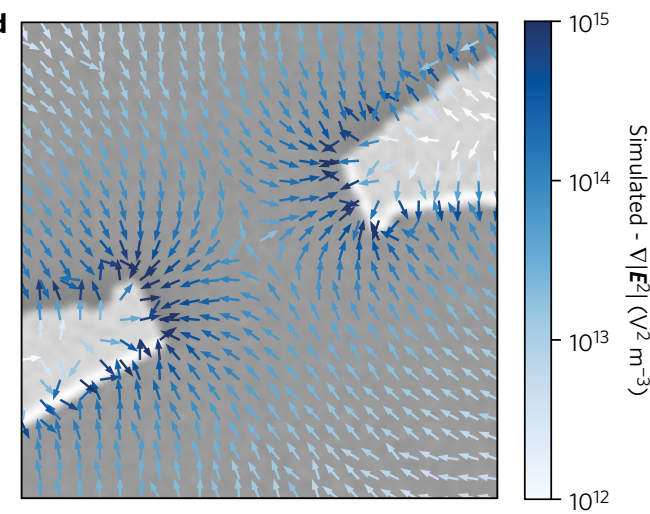

e
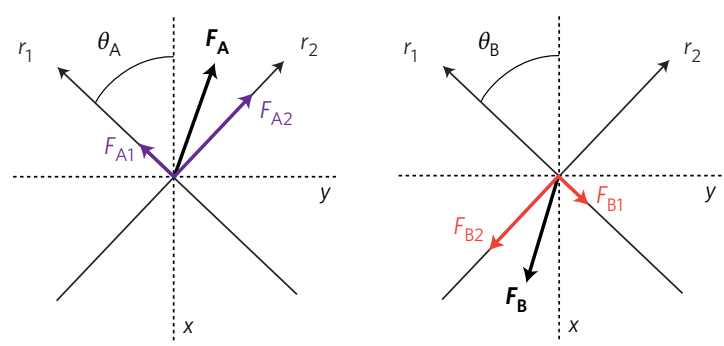

Figure 4 | Vector plots of electrostatic force fields. a, Vector plot of the force field induced by an electric field on the charged NW. The vector plot is overlain on top of an SEM image of the sample. Scale bar, $1 \mu \mathrm{m}$. $\mathbf{b}$, Vector plot of the simulated electric field $\boldsymbol{E}(\boldsymbol{r})$ induced by the biased finger gates. c, Vector plot of the force field induced by the electric field on the polarizable NW. The voltage amplitudes applied to the gates in a,c are $2 \mathrm{mV}$ and $20 \mathrm{mV}$, respectively, the vertical spacing between NW2 and the Au gates is $70 \mathrm{~nm}$ and $\theta_{0}=46^{\circ}$. $\mathbf{d}$, Vector plot of the simulated values of $-\nabla|\boldsymbol{E}(\boldsymbol{r})|^{2}$ induced by the biased finger gates. $\mathbf{e}$, Schematic explanation of the method used to extract force vectors from the measurements at the example positions $A$ and $B$ shown in $\mathbf{c}$. Example plots on the right showing the amplitude and phase responses as a function of the driving frequency illustrate the method (violet/red for positions A/B). The magnitude of the force component along each mode direction is extracted from the displacement of that mode. The phase response of the mode with respect to the driving then allows the extraction of the sign of the force component along each mode direction (see Methods section).

couple the two flexural modes and rotate their oscillation direction along two new basis vectors $\hat{\boldsymbol{r}}_{i}^{30}$. These new modes remain orthogonal for conservative force fields (that is, for $F_{12}=F_{21}$ ), but lose their orthogonality for non-conservative force fields.

For tip-sample force derivatives that are much smaller than the bare NW spring constant-which is the case here-the modified spring constants $k_{i}^{\prime} \approx k_{i}-F_{i i}$. Therefore, by monitoring the frequency shift between the bare resonances and those modified by the tip-sample interaction $\Delta f_{i}=f_{i}^{\prime}-f_{i}$, we measure:

$$
\left.\frac{\partial F_{i}}{\partial r_{i}}\right|_{0} \approx-2 k_{i}\left(\frac{\Delta f_{i}}{f_{i}}\right)
$$

Because the oscillation amplitude (6 nm) is small compared with the tip diameter (around $350 \mathrm{~nm}$ ), we can ignore spatial variations of the force derivative over the oscillation cycle. Just as in conventional 1D dynamic lateral force microscopy, each $\Delta f_{i}(x, y)$ depends on the derivative $F_{i i}$, that is, on the force both projected and differentiated along the mode oscillation direction. The NW mode doublet, however, is able to simultaneously measure force derivatives along orthogonal directions.

In the same limit of small derivatives considered for equation (3), the rotation of the mode axes reveals the shear, or cross-derivatives of the force, through,

$$
\left.\frac{\partial F_{i}}{\partial r_{j}}\right|_{0} \approx\left|k_{i}-k_{j}\right| \tan \phi_{i}
$$

for $i \neq j$ and where $\phi_{i}$ is the angle between the mode direction $\hat{\boldsymbol{r}}_{i}^{\prime}$ in the presence of tip-surface interaction and the bare mode direction $\hat{\boldsymbol{r}}_{i}$. Therefore by monitoring the doublet mode frequency shifts and oscillation directions, we can completely determine the in-plane tip-sample force derivatives $F_{i j}$. 
Imaging static in-plane force derivatives and dissipation A complete measurement of static in-plane force derivatives, the resulting force field and dissipations are shown in Fig. 3 for a section of the 'finger' sample using a second NW, NW2. $\Delta f_{i}$ and $\Gamma_{i}$ are extracted from fits to the spectral density of the thermal noise measured as the sample is scanned below the NW at a fixed spacing of $70 \mathrm{~nm}$. By assuming a conservative tip-sample interaction $\left(F_{i j}=F_{j i}\right)$, we determine $\phi=\phi_{1}=\phi_{2}$ from the frequency and integrated power of each mode in the spectral density compared with a similar measurement far from the surface, which results in $\theta_{0}=46^{\circ}$. To measure any force field, including nonconservative force fields such as those due to optomechanical interactions ${ }^{22}, \phi_{1}$ and $\phi_{2}$ have to be measured independently. This task requires a displacement measurement that is capable of determining both the direction and magnitude. Although not demonstrated here, such a measurement is possible in this set-up by carefully tuning the alignment of the interferometer with respect to the central axis of the $\mathrm{NW}^{22,29}$.

Using these data along with equations (3) and (4), we produce maps of $F_{i j}(x, y)$ and $\Gamma_{i}(x, y)$. The measurements show strong positive followed by negative $F_{i i}$ for edges perpendicular to the NW mode oscillation $\hat{\boldsymbol{r}}_{i}$. In non-contact AFM, tip-samples forces are generally attractive and become more so with decreasing tip-sample distance and increased interaction area. As the NW approaches an Au edge that is perpendicular to $\hat{\boldsymbol{r}}_{i}$ from a position above the lower $\mathrm{Si}$ surface, it experiences an increasingly attractive force- that is, a positive $F_{i i}$. After the midpoint of the tip crosses this edge, the attractive force starts to drop off, resulting in a negative $F_{i i}$. By integrating the force derivatives, we produce a map of the static in-plane force field due to tip-sample interactions $\boldsymbol{F}_{\text {int }}$, shown in Fig. 3e. Note that this force field is determined up to an integration constant, that is, up to a spatially constant force. Measured dissipations $\Gamma_{i}$ in Fig. 3f,g are nearly isotropic and appear to reflect the different materials and tip-sample spacings over the Au fingers and the Si substrate. Similar 1D measurements of non-contact friction also show lower values over conducting surfaces such as Au compared with insulating surfaces such as $\mathrm{Si}$ and point to charge fluctuations as the origin for the dissipation ${ }^{21,31}$.

\section{Imaging a dynamic in-plane force field}

In-plane force fields can also be mapped with a thermally limited sensitivity of $5 \mathrm{aN} \mathrm{Hz}^{-1 / 2}$ by measuring the driven NW response, as shown in Fig. 4. The frequency of the force to be measured must be swept through the NW doublet resonances, while the amplitude and phase of the displacement response is recorded. As an example, we apply a small a.c. voltage $V \cos (2 \pi f t)$ with frequency $f$ producing an oscillating electric field $\boldsymbol{E}(\boldsymbol{r}, t)$ between opposing finger gates. In scanning the NW and measuring its response, we distinguish between two types of forces. The first shows a linear dependence on the electric field strength and is associated with charge: $\boldsymbol{F}_{\mathrm{q}}=q \boldsymbol{E}$, where $q$ is the net charge on the NW tip. The second force exhibits a quadratic dependence on the field strength and is associated with the induced dipolar moment of the dielectric NW, that is, with its polarizability: $\boldsymbol{F}_{\mathrm{p}}=-\nabla\left(\alpha|\boldsymbol{E}|^{2}\right)$, where $\alpha$ is the effective polarizability of the GaAs/AlGaAs $\mathrm{NW}^{32}$. Owing to their linear and quadratic dependence on $\boldsymbol{E}$, respectively, $\boldsymbol{F}_{\mathrm{q}}$ drives the $\mathrm{NW}$ at frequency $f$, while $\boldsymbol{F}_{\mathrm{p}}$ drives it at d.c. and $2 f$. As a result, the two interactions can be spectrally separated.

The magnitude and orientation of the driving forces along each mode direction can be extracted from the displacement and phase response, respectively, of each mode as a function of frequency, as shown schematically in Fig. 4e. A measurement of the thermal noise spectrum in the absence of the a.c. drive is used to calibrate the orientation of the mode doublet. By scanning the sample in a plane $70 \mathrm{~nm}$ below NW2 and measuring the thermal motion and driven response at each point, we construct vectorial maps of $\boldsymbol{F}_{\mathrm{q}}$ and $\boldsymbol{F}_{\mathrm{p}}$, as shown in Fig. 4a,c and described in the Methods section. These measured force fields are compared to the $\boldsymbol{E}$ and $-\nabla\left|\boldsymbol{E}^{2}\right|$ fields simulated by finite element modelling analysis (COMSOL) using the real gate geometries in Fig. $4 \mathrm{~b}$,d. Dividing the measured force fields by the corresponding simulations we determine the net charge on the NW tip $q=30 \pm 10 e$, where $e$ is the fundamental charge and the effective polarizability $\alpha=10^{-29} \mathrm{CV}^{-1} \mathrm{~m}^{-1}$, which is roughly consistent with the size and dielectric constant of the NW. The ability to vectorially map electric fields on the nanometre scale extends the capability of conventional AFM to image charges ${ }^{33,34}$ and contact potential differences ${ }^{20}$ and has applications in localizing electronic defects on or near surfaces, for example, in microelectronic failure analysis.

\section{Conclusions}

Our measurements demonstrate the potential of NWs as sensitive scanning vectorial force sensors. By monitoring two orthogonal flexural modes while scanning over a sample surface, we map the forces and force derivatives in $2 \mathrm{D}$. Although we demonstrate the technique on electrostatic tip-sample interactions, it is generally applicable and could be used, for instance, to measure magnetic forces with proper functionalization of the NW tip or even in liquid ${ }^{35}$ for the study of batteries, water splitting or fuel cells. Note that in the data presented here, the diameter of the NW tip $(350 \mathrm{~nm})$ strongly limits the spatial resolution of the microscopy. Nevertheless, the same directional technique could be applied to NWs that are grown or processed to have sharp tips, presenting the possibility of atomicscale or even subatomic-scale force microscopy, a feat already achieved in $1 \mathrm{D}^{27}$. Therefore, one could imagine the use of vectorial NW-based AFM of tip-sample forces and non-contact friction to reveal, for example, the anisotropy of atomic bonding forces.

\section{Methods}

Methods and any associated references are available in the online version of the paper.

Received 1 April 2016; accepted 24 August 2016; published online 17 October 2016

\section{References}

1. Binnig, G., Quate, C. F. \& Gerber, C. Atomic force microscope. Phys. Rev. Lett. 56, 930-933 (1986).

2. Giessibl, F. J. Advances in atomic force microscopy. Rev. Mod. Phys. 75, 949-983 (2003).

3. Giessibl, F. J. Atomic resolution of the silicon $(111)-(7 \times 7)$ surface by atomic force microscopy. Science 267, 68-71 (1995).

4. Giessibl, F. J., Hembacher, S., Bielefeldt, H. \& Mannhart, J. Subatomic features on the silicon (111)-(7×7) surface observed by atomic force microscopy. Science 289, 422-425 (2000).

5. Poggio, M. Sensing from the bottom up. Nat. Nanotech. 8, 482-483 (2013).

6. Sazonova, V. et al. A tunable carbon nanotube electromechanical oscillator. Nature 431, 284-287 (2004).

7. Bunch, J. S. et al. Electromechanical resonators from graphene sheets. Science 315, 490-493 (2007).

8. Perisanu, S. et al. High $Q$ factor for mechanical resonances of batch-fabricated SiC nanowires. Appl. Phys. Lett. 90, 043113 (2007).

9. Feng, X. L., He, R., Yang, P. \& Roukes, M. L. Very high frequency silicon nanowire electromechanical resonators. Nano Lett. 7, 1953-1959 (2007).

10. Nichol, J. M., Hemesath, E. R., Lauhon, L. J. \& Budakian, R. Displacement detection of silicon nanowires by polarization-enhanced fiber-optic interferometry. Appl. Phys. Lett. 93, 193110 (2008).

11. Li, M. et al. Bottom-up assembly of large-area nanowire resonator arrays. Nat. Nanotech. 3, 88-92 (2008).

12. Belov, M. et al. Mechanical resonance of clamped silicon nanowires measured by optical interferometry. J. Appl. Phys. 103, 074304 (2008).

13. Gil-Santos, E. et al. Nanomechanical mass sensing and stiffness spectrometry based on two-dimensional vibrations of resonant nanowires. Nat. Nanotech. 5, 641-645 (2010).

14. Chaste, J. et al. A nanomechanical mass sensor with yoctogram resolution. Nat. Nanotech. 7, 301-304 (2012).

15. Moser, J. et al. Ultrasensitive force detection with a nanotube mechanical resonator. Nat. Nanotech. 8, 493-496 (2013). 
16. Moser, J., Eichler, A., Güttinger, J., Dykman, M. I. \& Bachtold, A. Nanotube mechanical resonators with quality factors of up to 5 million. Nat. Nanotech. 9, 1007-1011 (2014).

17. Gysin, U., Rast, S., Kisiel, M., Werle, C. \& Meyer, E. Low temperature ultrahigh vacuum noncontact atomic force microscope in the pendulum geometry. Rev. Sci. Instrum. 82, 023705 (2011).

18. Nichol, J. M., Hemesath, E. R., Lauhon, L. J. \& Budakian, R. Nanomechanical detection of nuclear magnetic resonance using a silicon nanowire oscillator. Phys. Rev. B 85, 054414 (2012).

19. Nichol, J. M., Naibert, T. R., Hemesath, E. R., Lauhon, L. J. \& Budakian, R. Nanoscale fourier-transform magnetic resonance imaging. Phys. Rev. X 3, 031016 (2013).

20. Nonnenmacher, M., O’Boyle, M. P. \& Wickramasinghe, H. K. Kelvin probe force microscopy. Appl. Phys. Lett. 58, 2921-2923 (1991).

21. Stipe, B. C., Mamin, H. J., Stowe, T. D., Kenny, T. W. \& Rugar, D. Noncontact friction and force fluctuations between closely spaced bodies. Phys. Rev. Lett. 87, 096801 (2001).

22. Gloppe, A. et al. Bidimensional nano-optomechanics and topological backaction in a non-conservative radiation force field. Nat. Nanotech. 9, 920-926 (2014).

23. Pfeiffer, O., Bennewitz, R., Baratoff, A., Meyer, E. \& Grütter, P. Lateral-force measurements in dynamic force microscopy. Phys. Rev. B 65, 161403 (2002).

24. Giessibl, F. J., Herz, M. \& Mannhart, J. Friction traced to the single atom. Proc. Natl Acad. Sci. USA 99, 12006-12010 (2002).

25. Kawai, S., Kitamura, S.-I., Kobayashi, D. \& Kawakatsu, H. Dynamic lateral force microscopy with true atomic resolution. Appl. Phys. Lett. 87, 173105 (2005)

26. Kawai, S., Sasaki, N. \& Kawakatsu, H. Direct mapping of the lateral force gradient on Si (111)-(7×7). Phys. Rev. B 79, 195412 (2009).

27. Kawai, S. et al. Ultrasensitive detection of lateral atomic-scale interactions on graphite (0001) via bimodal dynamic force measurements. Phys. Rev. B 81, 085420 (2010).

28. Cadeddu, D. et al. Time-resolved nonlinear coupling between orthogonal flexural modes of a pristine GaAs nanowire. Nano Lett. 16, 926-931 (2016)

29. Karabacak, D., Kouh, T., Huang, C. C. \& Ekinci, K. L. Optical knife-edge technique for nanomechanical displacement detection. Appl. Phys. Lett. 88, 193122 (2006)

30. Faust, T. et al. Nonadiabatic dynamics of two strongly coupled nanomechanical resonator modes. Phys. Rev. Lett. 109, 037205 (2012).
31. Kuehn, S., Loring, R. F. \& Marohn, J. A. Dielectric fluctuations and the origins of noncontact friction. Phys. Rev. Lett. 96, 156103 (2006).

32. Rieger, J., Faust, T., Seitner, M. J., Kotthaus, J. P. \& Weig, E. M. Frequency and $\mathrm{Q}$ factor control of nanomechanical resonators. Appl. Phys. Lett. 101, 103110 (2012).

33. Stern, J. E., Terris, B. D., Mamin, H. J. \& Rugar, D. Deposition and imaging of localized charge on insulator surfaces using a force microscope. Appl. Phys. Lett. 53, 2717-2719 (1988).

34. Schönenberger, C. \& Alvarado, S. F. Observation of single charge carriers by force microscopy. Phys. Rev. Lett. 65, 3162-3164 (1990).

35. Sanii, B. \& Ashby, P. D. High sensitivity deflection detection of nanowires. Phys. Rev. Lett. 104, 147203 (2010).

\section{Acknowledgements}

We thank S. Martin and the mechanical workshop at the University of Basel Physics Department for help in designing and building the NW microscope and J. Teissier for useful discussions. We acknowledge the support of the ERC through Starting Grants NWScan (Grant No. 334767) and UpCon (Grant No. 239743), the Swiss Nanoscience Institute (Project P1207), the Swiss National Science Foundation (Ambizione Grant No. PZ00P2-161284/1 and Project Grant No. 200020-159893) and the NCCR Quantum Science and Technology (QSIT).

\section{Author contributions}

N.R. and F.R.B. performed the experiment, G.T. and A.F.i.M. grew the nanowires, D.V., N.R., D.C. and M.P. designed and constructed the measurement set-up. N.R. fabricated the sample. N.R. and F.R.B. undertook the data analysis. N.R., F.R.B., and M.P. contributed to the interpretation of the data and wrote the manuscript. All authors commented and contributed to the manuscript. M.P. conceived and supervised the project.

\section{Additional information}

Supplementary information is available in the online version of the paper. Reprints and permissions information is available online at www.nature.com/reprints. Correspondence and requests for materials should be addressed to M.P.

\section{Competing financial interests}

The authors declare no competing financial interests. 


\section{Methods}

Nanowire growth and processing. Molecular beam epitaxy synthesis of the GaAs/AlGaAs NWs starts on a Si substrate with the growth of a 290 -nm-thick GaAs NW core along [11ㅣ] by the Ga-assisted method detailed in Uccelli et al. ${ }^{36}$ and Russo-Averchi and colleagues ${ }^{37}$. Axial growth is stopped once the NWs are about $25 \mu \mathrm{m}$ long by temporarily blocking the $\mathrm{Ga}$ flux and reducing the substrate temperature from $630^{\circ} \mathrm{C}$ down to $465^{\circ} \mathrm{C}$. Finally, a 50 -nm-thick $\mathrm{Al}_{0.51} \mathrm{Ga}_{0.49}$ As shell capped by a $5 \mathrm{~nm} \mathrm{GaAs}$ layer is grown ${ }^{38}$. For optimal inferometric detection of the NWs, we focus on NWs within $40 \mu \mathrm{m}$ of the substrate edge. To avoid measuring multiple NWs or interference between NWs, we reduce the NW density in the area of interest using a micromanipulator under an optical microscope.

Nanowire positioning and displacement detection. The measurement chamber includes two stacks of piezoelectric positioners (Attocube AG) to independently control the 3D position of the NW cantilevers and the sample of interest with respect to the fixed detection optics. The top stack is used to align a single NW within the focus of a fibre-coupled optical interferometer used to detect its mechanical motion $^{39}$. Once the NW and interferometer are aligned, the bottom stack is used to approach and scan the sample of interest with respect to the NW cantilever. Light from a laser diode with wavelength of $635 \mathrm{~nm}$ is sent through one arm of a 50:50 fibre-optic coupler and focused by a pair of lenses to a $1 \mu \mathrm{m}$ spot. The incident power of around $5 \mu \mathrm{W}$ does not significantly heat the NW as confirmed by measurements of laser power dependence and mechanical thermal motion. Despite the submicrometre diameter of the $\mathrm{NW}$, it reflects a portion of the light back into the fibre, which interferes with light reflected by the cleaved end. The resulting low-finesse Fabry-Perot interferometer acts as a sensitive sensor of the NW displacement, where the interference intensity is measured by a fast photoreceiver with an effective $3 \mathrm{~dB}$ bandwidth of $800 \mathrm{kHz}$.

Measurement protocols. Measurements of the NW thermal motion used for Figs 1, 3 and 4 are made using an analog-to-digital converter (National Instruments). Fits based on the thermally driven damped harmonic oscillator of equation (1) are used to extract $\theta_{0}, f_{i}, \Gamma_{i}, m$, and $\phi_{i}$. The consideration of an instrumental mechanical vibration between the sample and the NW is required in these fits to produce integrable force derivatives. The root-mean-squared amplitude of this vibration is $3 \mathrm{~nm}$, which is reasonable for our experimental set-up. This parameter only affects measurements of $F_{12}=F_{21}$ in Fig. $3 \mathrm{c}$, which depend on $\phi_{i}$ and are derived from the ratio of the mean square displacements of the two modes. Faster measurements, as in Fig. 2, are performed by resonantly exciting both of the flexural modes using the piezoelectric transducer. Owing to the high $Q_{i}$ and large $\delta$ of the resonances, it is possible to monitor and control both modes simultaneously. As in standard AFM, we use a lockin amplifier (Zurich Instruments UHFLI) to resonantly drive each mode and demodulate the resulting optical signal measured by the photoreceiver. For each mode, its shift in resonant frequency $\Delta f_{i}$ is tracked by a phase-locked loop, while proportional integral control of the excitation voltage maintains a constant oscillation amplitude. Measurements of the NW amplitude and phase response to a time-varying gate voltage applied to the sample, as shown in Fig. 4, are made using the same lock-in. Fits to the amplitude and phase response of each mode as a function of frequency are based on the mechanical susceptibility $\chi_{i}(\omega)=1 /\left(k_{i}-m \omega^{2}+i \Gamma_{i} \omega\right)$ of a damped harmonic oscillator, where $\omega=2 \pi f$. The oscillation amplitude

$\left|\chi_{i}(\omega)\right|\left|F_{i}(\omega)\right|$ excited for mode $i$ gives the magnitude of the driving force $F_{i}$ along $\hat{\boldsymbol{r}}_{i}$. The phase $\arg \chi_{i}(\omega)+\arg F_{i}(\omega)$ gives the orientation of $F_{i}$ along either positive or negative $\hat{\boldsymbol{r}}_{i}$.

\section{References}

36. Uccelli, E. et al. Three-dimensional multiple-order twinning of self-catalyzed GaAs nanowires on Si substrates. Nano Lett. 11, 3827-3832 (2011).

37. Russo-Averchi, E. et al. Suppression of three dimensional twinning for a $100 \%$ yield of vertical GaAs nanowires on silicon. Nanoscale 4, 1486-1490 (2012).

38. Heigoldt, M. et al. Long range epitaxial growth of prismatic heterostructures on the facets of catalyst-free GaAs nanowires. J. Mater. Chem. 19, 840-848 (2009).

39. Rugar, D., Mamin, H. J. \& Guethner, P. Improved fiber-optic interferometer for atomic force microscopy. Appl. Phys. Lett. 55, 2588-2590 (1989). 